\title{
Competencias digitales de docentes de nivel secundario de Santo Domingo: un estudio de caso
}

\author{
Digital Competences of Secondary Level of Santo Domingo: A Case Study
}

Habilidades digitais de professores do ensino médio em Santo Domingo: um estudo de caso

José Antonio Vólquez Pérez

Universidad de Baja California, México

volquez.jose@gmail.com

https://orcid.org/0000-0002-0518-0181

Carlos Miguel Amador Ortíz

Instituto Tecnológico José Mario Molina Pasquel y Henríquez, Campus Puerto Vallarta,

México

carlos.amador@vallarta.tecmm.edu.mx https://orcid.org/0000-0001-6654-8448

\section{Resumen}

En los últimos años se ha generado una transformación en la educación a causa del uso de las tecnologías de la comunicación y de la información (TIC). A partir de esta transformación se espera que los docentes desarrollen nuevos conocimientos y habilidades para el uso de la tecnología. Este trabajo tiene como propósito analizar el uso de las TIC en el proceso de enseñanza-aprendizaje por parte de maestros de nivel secundario con el fin de establecer estrategias para el desarrollo de competencias digitales orientadas a la docencia. Para esto se utilizó una metodología descriptiva, analítica y transversal, y se aplicó un cuestionario con una escala de valoración de competencias digitales considerando tres dimensiones: competencias instrumentales, competencias didáctico-metodológicas y cognitivas. Este cuestionario se aplicó a un total de 124 docentes de dos instituciones de educación secundaria del distrito 10-04 de Santo Domingo, República Dominicana. Los resultados muestran que $47 \%$ de los docentes 


\section{Resumo}

Nos últimos anos, uma transformação foi gerada na educação devido ao uso das tecnologias da comunicação e informação (TIC). A partir dessa transformação, espera-se que os professores desenvolvam novos conhecimentos e habilidades para o uso da tecnologia. O objetivo deste trabalho é analisar o uso das TIC no processo de ensinoaprendizagem por professores do ensino médio, a fim de estabelecer estratégias para o desenvolvimento de habilidades digitais orientadas ao ensino. Para isso, utilizou-se uma metodologia descritiva, analítica e transversal, e aplicou-se um questionário com escala para avaliação de habilidades digitais, considerando três dimensões: habilidades instrumentais, habilidades didático-metodológicas e cognitivas. Este questionário foi aplicado a um total de 124 professores de duas instituições de ensino médio no distrito 10-04 de Santo Domingo, República Dominicana. Os resultados mostram que 47\% dos professores necessitam de treinamento em competências didático-metodológicas digitais, $39 \%$ em cognitiva e $32 \%$ em instrumental. A partir desses resultados, conclui-se com a necessidade de estabelecer um plano de treinamento que considere prioritárias as competências nas quais os menores resultados foram obtidos.

Palavras-chave: formação de professores, integração, processos de ensinoaprendizagem, recursos didáticos, TIC.

Fecha Recepción: Febrero 2020

Fecha Aceptación: Julio 2020

\section{Introducción}

La transmisión de conocimientos ha sido un factor determinante en el desarrollo de la civilización humana: desde los fundamentos de supervivencia en sus inicios hasta la formación especializada que requiere una sociedad industrial con alrededor de 6000 millones de habitantes. Es debido a la importancia de la transmisión de conocimientos, sobre todo con las demandas de una sociedad diversa e industrializada, que el tema educativo adquiere protagonismo para muchos intelectuales, y es un área en constante desarrollo, ya que las prácticas formativas deben estar en constante actualización con el fin de formar a los individuos y generar sociedades con diversos grados de tecnificación y especialización.

Dado al ajuste de las prácticas a las demandas de la modernidad, el maestro no se puede quedar rezagado, su nuevo rol en los centros educativos requiere no solo el conocimiento académico, sino también el manejo práctico de la tecnología, y su 


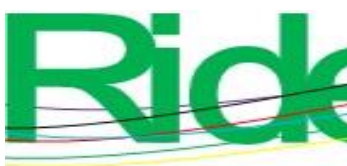

Revista Iberoamericana para la Investigación y el Desarrollo Educativo ISSN $2007-7467$

aplicación como herramienta esencial en el proceso de enseñanza-aprendizaje. Las tecnologías de la información y comunicación (TIC) han generado un impacto y una serie de cambios en todos los ámbitos de la sociedad. El manejo y uso de las TIC es fundamental en la práctica pedagógica.

Por tanto, el profesor tendría que superar la aplicación de procedimientos y visiones de la enseñanza tradicionales e impersonales que no reconocen al alumno como protagonista del proceso. Las nuevas necesidades educativas del siglo XXI ya no pueden adaptarse al uso de una metodología rígida, terminada e intransigente, y que no toman en cuenta las experiencias del alumno y su necesidad de comprender la realidad (Reis y Lunardi, 2018; Silveira y de Luca, 2015; Zabala, 2000). Así pues, corresponde al maestro reconocer las necesidades de los estudiantes y crear mecanismos que puedan satisfacer a estos durante su práctica diaria (Turra y Flores, 2018).

Existen diferentes factores que inciden en el desarrollo de competencias digitales por parte de los docentes. Uno de ellos es la brecha generacional. Los inmigrantes digitales, de acuerdo con Prensky (2012), son aquellos que nacieron antes de la revolución de las TIC y su apropiación ha sido difícil, o de poco interés, ya que su base de estudios profesionales se desarrolló sin estas herramientas. Otro de los factores es la falta de acceso a las tecnologías y la falta de capacitación en competencias para el uso de las tecnologías con enfoque específico para los procesos formativos.

Integrar las TIC en el sistema educativo tiene sus implicaciones, entre ellas, la falta de interés y la poca preparación de los docentes para introducirlas en su práctica en el aula (Fernández y Fernández, 2016); además, hacen falta proyectos que fomenten la utilización de las TIC en los centros escolares; por tanto, muchas de las prácticas didácticas que se realizan con las tecnologías digitales no representan una verdadera innovación que impacte de manera directa en los procesos de enseñanza y aprendizaje (Christian y Mathrani, 2014).

Las normas propias de los centros escolares pueden dificultar la implementación de las TIC en las instituciones educativas; la organización y cultura de la escuela pueden obstaculizar los procesos de innovación con el uso de las TIC. Esto puede tener como consecuencia que no se produzcan los cambios que auguraba la integración de las TIC en las aulas (Alonso et al., 2010; Medina y Ballano, 2015).

Franky y Chiappe (2018) mencionan que, aunque la educación está cada vez más impregnada de tecnologías, actualmente no es capaz de responder a los desafíos de nuestros tiempos. Esta época se caracteriza por un gran dinamismo y cambio social. La democratización de la información, los cambios en la estructura de las familias, los 
medios de comunicación y las redes sociales están configurando una sociedad diversa con demandas educativas que la tecnología por sí misma no es capaz de satisfacer. Las tecnologías han revolucionado los procesos de aprendizaje, y se presentan como herramientas muy valiosas por su potencial para mejorar los procesos formativos y por la diversidad de herramientas para adquirir conocimientos, inclusive hacen posible la formación a distancia a través de las plataformas online, lo que puede resolver diversas problemáticas presentadas en la educación tradicional; sin embargo, estas deben estar integradas dentro de un sistema educativo sistematizado y funcional orientado a las necesidades sociales de formación para poder responder a los desafíos que imperan hoy en día.

Presionadas por una sociedad tecnológica e informativa, las instituciones educativas invierten grandes recursos para adquirir infraestructuras tecnológicas con el fin de aumentar la calidad de su educación. Pese a ello, siguiendo a ciertos autores, los resultados obtenidos no parecen justificar esta inversión (Christensen, Johnson y Horn, 2008; Sosa y Mazuolli, 2019). En esta misma línea, Rincón (2018) menciona que un aumento en la infraestructura tecnológica no implica directamente un cambio en la manera de enseñar.

Otros autores presentan una visión diferente. Enguita (2016), por ejemplo, afirma que la sociedad contemporánea puede identificarse como una sociedad de cambio. Este no es un cambio trivial y común, como lo ha sido en la historia, sino un cambio social muy rápido nunca antes visto. En este cambio, el uso de la tecnología es un componente esencial aplicado en diferentes ámbitos, tal es el caso de las inversiones para adquirir infraestructura tecnológica por parte de las instituciones educativas, lo cual tiene que ir acompañado de un plan de capacitación para los docentes que permita aprovechar las ventajas de estas herramientas para responder a los desafíos de nuestros tiempos.

Esta sociedad de cambio conlleva nuevos escenarios. Los roles tradicionales del maestro como portador y fuente de transmisión de conocimientos pierden su preponderancia, ya que se propicia el aprendizaje por mediación de tecnologías (como fuentes de conocimiento y herramientas para el aprendizaje).

Para Cabero, Arancibia, Valdivia y Aranedas (2018), existe una actitud positiva tanto en los docentes como en los estudiantes para utilizar herramientas formativas virtuales en su proceso de aprendizaje, lo cual facilita la integración de estas en las instituciones educativas. Además de esto, Jiménez y Espejel (2019) mencionan que se está considerando en la agenda de las políticas públicas la integración de las TIC en los 


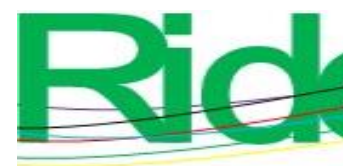

Revista Iberoamericana para la Investigación y el Desarrollo Educativo ISSN 2007 - 7467

sistemas educativos, tanto en México como en América Latina, con la finalidad de que el estudiante pueda desarrollar habilidades para su desempeño académico y laboral.

El docente es considerado un agente clave y mediador de las prácticas educativas y tecnológicas en los diferentes enfoques del uso de las TIC en el ámbito educativo (Rubilar, Alveal y Fuentes, 2017); no obstante, aunque exista una buena actitud para el uso de las TIC por parte de ellos, en la actualidad no se sienten competentes y evitan utilizarlas (Ayala, 2018). De allí la importancia de promover estrategias que contribuyan al cambio y a la adaptación a los nuevos escenarios y roles que implica la utilización de las TIC en el aula, y de mantener una capacitación constante sobre el uso de la tecnología para la innovación de la práctica educativa como un elemento indispensable para lograr los resultados esperados de su implementación.

En la presente investigación se acoge el modelo de Quintana (2000), quien especifica las destrezas en los estándares de competencia en la utilización de las TIC para docentes, los cuales han sido propuestos por la Organización de las Naciones Unidas para la Educación, la Ciencia y la Cultura (Unesco) en el año 2008, la cual, además, ha definido las competencias de tipo técnico (saber), de tipo metodológico (saber hacer) y de tipo social (saber ser).

Según la Comisión Europea (30 de diciembre de 2006), la competencia digital “entraña el uso seguro y crítico de las tecnologías de la sociedad de la información para el trabajo, el ocio y la comunicación " (p. 6), y señala que para el desarrollo de estas se necesita contar con un adecuado entendimiento y un saber amplio sobre "la naturaleza, la función y las oportunidades de las tecnologías de la sociedad de la información en situaciones cotidianas de la vida privada, social y profesional" (p. 7).

Para Quintana (2000), la incorporación de las TIC en los procesos de enseñanza y aprendizaje requiere del desarrollo de tres categorías de competencias digitales: 1) competencias digitales instrumentales, dirigidas al saber y a la utilización tanto del equipo como del programa informático para el desarrollo del proceso educativo en la indagación, adquisición y del procesamiento de la información; 2) competencias digitales cognitivas, que involucran la reflexión y la aplicación de un criterio sobre la utilización de las TIC dentro del aprendizaje, y 3) competencias digitales didácticas-metodológicas, fundamentadas en el acoplamiento de las TIC dentro del proceso de enseñanza y aprendizaje en el salón de clases, de forma que facilite la elaboración o diseño de la unidad didáctica, así como de la actividad de aprendizaje. En la tabla 1 se pueden observar los indicadores de las categorías. 
Tabla 1. Indicadores de las categorías de competencias digitales

\begin{tabular}{|c|c|c|}
\hline $\begin{array}{l}\text { Competencias digitales } \\
\text { instrumentales }\end{array}$ & $\begin{array}{l}\text { Competencias didácticas- } \\
\text { metodológicas }\end{array}$ & $\begin{array}{l}\text { Competencias cognitivas- } \\
\text { actitudinales }\end{array}$ \\
\hline $\begin{array}{l}\text { Conocimiento y empleo de } \\
\text { aparatos informáticos }\end{array}$ & $\begin{array}{l}\text { Utiliza herramientas } \\
\text { tecnológicas para preparar } \\
\text { sus clases, para seguir el } \\
\text { proceso de aprendizaje } \\
\text { logrando evaluar al } \\
\text { estudiante, gestión } \\
\text { académica, su formación } \\
\text { permanente, y participar en } \\
\text { proyectos con otros docentes } \\
\text { de la institución. }\end{array}$ & $\begin{array}{l}\text { Aplica criterios en el uso de } \\
\text { las tecnologías. }\end{array}$ \\
\hline $\begin{array}{l}\text { Conocimiento y uso } \\
\text { funcional y creativo de los } \\
\text { programas informáticos } \\
\text { instrumentales estándar } \\
\text { (entorno operativo, } \\
\text { procesador de textos, base } \\
\text { de datos, hoja de cálculo, } \\
\text { etc.) }\end{array}$ & $\begin{array}{l}\text { Evalúa o selecciona } \\
\text { programas informáticos o en } \\
\text { línea. }\end{array}$ & $\begin{array}{l}\text { Posee actitudes de reflexión } \\
\text { sobre los usos de los medios } \\
\text { en el aprendizaje y en la } \\
\text { educación en general, y } \\
\text { sobre la propia actividad } \\
\text { como maestros y maestras. }\end{array}$ \\
\hline $\begin{array}{l}\text { Conocimiento y uso } \\
\text { funcional y creativo de los } \\
\text { programas informáticos } \\
\text { estándar para la educación }\end{array}$ & $\begin{array}{l}\text { Crea unidades de } \\
\text { aprendizaje incorporando las } \\
\text { TIC. }\end{array}$ & $\begin{array}{l}\text { Utiliza habilidades para el } \\
\text { tratamiento de la } \\
\text { información: análisis e } \\
\text { interpretación (evaluación, } \\
\text { comparación, contraste, } \\
\text { elaboración, representación, } \\
\text { relación, síntesis, } \\
\text { valoración), uso y } \\
\text { comunicación (aplicación, } \\
\text { asimilación, expresión, } \\
\text { integración, presentación, } \\
\text { transferencia). }\end{array}$ \\
\hline
\end{tabular}




\begin{tabular}{|l|l|l|}
\hline $\begin{array}{l}\text { Habilidades para el } \\
\text { tratamiento de la } \\
\text { información }\end{array}$ & $\begin{array}{l}\text { Integra las TIC en los } \\
\text { procesos de aprendizaje } \\
\text { cotidianos en el aula. }\end{array}$ & \\
\hline & $\begin{array}{l}\text { Utiliza las TIC para facilitar } \\
\text { la comunicación y atender a } \\
\text { la diversidad. }\end{array}$ & \\
\hline
\end{tabular}

Fuente: Elaboración propia con base en Quintana (2000)

El propósito de este estudio fue analizar el uso de las TIC en el proceso de enseñanza-aprendizaje por parte de maestros de nivel secundario, con el fin de establecer estrategias para el desarrollo de competencias digitales orientadas a la docencia, tomando como referencia el modelo teórico de Quintana (2000), modelo que categoriza, como ya hemos visto, las competencias digitales en instrumentales, didáctico-metodológicas y cognitivo-actitudinales.

\section{Objetivo general}

Este estudio tuvo como objetivo general analizar el uso de las TIC en el proceso enseñanza-aprendizaje de maestros de nivel secundario, en específico del Politécnico Pilar Constanzo y de la Escuela María Marcia Compres de Vargas del distrito 10-04 de Santo Domingo, República Dominicana. Esto con el fin de establecer estrategias para el desarrollo de competencias digitales orientadas a la docencia.

\section{Objetivos específicos}

1) Adoptar una postura teórica para el análisis de competencias digitales en docentes.

2) Describir las competencias digitales de los docentes del Politécnico Pilar Constanzo y de la Escuela María Marcia Compres de Vargas del distrito 10-04 de Santo Domingo Este, con base en una postura teórica reconocida por la comunidad académica.

3) Identificar áreas de oportunidad para el diseño de estrategias formativas para la profesionalización de los docentes.

4) Proponer estrategias para el desarrollo de competencias digitales de los docentes del Politécnico Pilar Constanzo y de la Escuela María Marcia Compres de Vargas del distrito 10-04 de Santo Domingo Este. 


\section{Materiales y métodos}

El presente estudio se basa en una metodología de investigación descriptiva, analítica y transversal, desarrollada mediante un enfoque cuantitativo en que se utilizó un cuestionario con una escala de valores, cuyas variables se cuantificaron. En el estudio se describe el desarrollo de competencias digitales de los docentes del Politécnico Pilar Constanzo y de la Escuela María Marcia Compres de Vargas, y se analizan los resultados con el fin de proponer estrategias para la integración de las TIC como recursos didácticos. La investigación es transversal, ya que se llevó a cabo una única vez.

El procedimiento que se siguió para el desarrollo de este trabajo corresponde a un método deductivo-inductivo, pues, además de partir de modelos preestablecidos (de lo general a lo particular), también se siguieron procesos inductivos en la recolección, análisis de los datos y en la formulación de conclusiones generales.

La investigación se desarrolló en las siguientes fases:

1) Investigación bibliográfica sobre competencias digitales en docentes, e instrumentos para analizar el uso de las TIC en la docencia.

2) Determinar el instrumento de recolección de información a utilizar.

3) Selección de la muestra y administración del instrumento.

4) Análisis de resultados.

\section{Investigación bibliográfica sobre competencias digitales en docentes, $\mathrm{e}$ instrumentos para analizar el uso de las TIC en la docencia}

A partir de la investigación bibliográfica se decidió adoptar como marco teóricoconceptual la propuesta de Quintana (2000) para el abordaje de las competencias digitales. Esta propone las siguientes categorías de competencias: digitales instrumentales, didáctico-metodológicas y cognitivo-actitudinales. Dichas categorías están conceptualizadas ya en el apartado introductorio de este trabajo, y los indicadores también ya aparecen descritos en la tabla 1 .

\section{Determinar el tipo de instrumento a administrar}

Asimismo, se utilizó un cuestionario elaborado por Espino (2018), el cual consta en total de 30 ítems, 10 ítems por cada una de las categorías adoptadas como marco teórico conceptual, y con una escala tipo Likert. A continuación, en la tabla 2, se describe la ficha técnica del instrumento. 
Tabla 2. Ficha técnica del instrumento utilizado

\begin{tabular}{|c|c|}
\hline \multicolumn{2}{|r|}{ Características } \\
\hline Nombre de instrumento & $\begin{array}{l}\text { Cuestionario sobre las competencias digitales de los } \\
\text { docentes }\end{array}$ \\
\hline Autor & Eugenio Espino Wuffarden \\
\hline Dirigido a & $\begin{array}{l}\text { Docentes del Instituto Politécnico Pilar Constanzo y de la } \\
\text { Escuela María Marcia Compres de Vargas de nivel } \\
\text { secundario del distrito 10-04, en Santo Domingo Este. }\end{array}$ \\
\hline Propósito & $\begin{array}{l}\text { Determinar el desarrollo de competencias digitales por } \\
\text { parte de los docentes de las escuelas Instituto Politécnico } \\
\text { Pilar Constanzo y María Marcia Compres de Vargas del } \\
\text { nivel secundario en el distrito 10-04. }\end{array}$ \\
\hline Forma de administración & Individual \\
\hline Dimensiones por evaluar & $\begin{array}{l}\text { - Dimensión 1: competencias digitales instrumentales } \\
\text { (10 ítems). } \\
\text { - Dimensión 2: competencias digitales didáctico- } \\
\text { metodológicas (10 ítems). } \\
\text { - Dimensión 3: competencias digitales cognitivas (10 } \\
\text { ítems). }\end{array}$ \\
\hline Escala de valoración & $\begin{array}{ll}\text { - } & \text { Siempre (5 puntos). } \\
\text { - } & \text { Casi siempre (4 puntos). } \\
\text { - } & \text { A veces ( } 3 \text { puntos). } \\
\text { - } & \text { Casi nunca ( } 2 \text { puntos). } \\
\text { - } & \text { Nunca (1 punto). }\end{array}$ \\
\hline
\end{tabular}

Fuente: Elaboración propia con base en Espino (2018)

Respecto a la validez del instrumento, se utilizaron los indicadores del marco conceptual propuesto por Quintana (2000), a partir del cual se elaboraron los ítems del cuestionario (ver tabla 1). Para la confiabilidad, se utilizó el coeficiente de consistencia interna llamado alfa de Cronbach. Como se puede observar en la tabla 3, se obtuvieron resultados altos en las tres dimensiones, lo que demuestra una fuerte relación entre los ítems de las categorías con base en las especificaciones de algunos autores como Celina y Campos (2005) y Lucero y Meza (2002). 
Tabla 3. Resultados de coeficiente de consistencia interna alfa de Cronbach

\begin{tabular}{|c|c|c|c|}
\hline Dimensión & Núm. de ítems & Alfa de Cronbach & Confiabilidad \\
\hline $\begin{array}{c}\text { Competencias } \\
\text { digitales } \\
\text { instrumentales }\end{array}$ & 10 & 0.926 & Alta \\
\hline $\begin{array}{c}\text { Competencias } \\
\text { digitales didáctico- } \\
\text { metodológicas }\end{array}$ & 10 & 0.925 & Alta \\
\hline $\begin{array}{c}\text { Competencias } \\
\text { digitales cognitivas }\end{array}$ & 10 & 0.925 & Alta \\
\hline Total & 30 & 0.928 & Alta \\
\hline
\end{tabular}

Fuente: Elaboración propia

Para la interpretación de los datos del cuestionario, Espino (2018) propone una escala basada en la sumatoria de las respuestas en cada una de las dimensiones con los datos que a continuación se describen:

- Bueno: de 40 a 50 puntos por dimensión, considerado nivel alto de desarrollo de competencias digitales, no requiere capacitación, pero sí estar en constante actualización de acuerdo con las demandas de los cambios tecnológicos.

- $\quad$ Regular: de 30 a 39 puntos por dimensión, considerado nivel medio que requiere de capacitación en algunas áreas.

- Deficiente: menos de 29 puntos por dimensión, nivel bajo que requiere de capacitación generalizada para el desarrollo de competencias digitales en la categoría en que se haya obtenido este puntaje.

\section{Seleccionar la muestra y administrar el instrumento}

El instrumento se administró al total de los docentes de nivel secundario de dos escuelas del distrito educativo 10-04 del municipio de Santo Domingo, el Politécnico Pilar Constanzo, que tiene 77 docentes, y la Escuela María Marcia Compres de Vargas, con 47 docentes. Así, se obtuvo una muestra de 124 docentes de este distrito.

\section{Análisis de resultados}

La información obtenida de los cuestionarios se procesó en una hoja de cálculo (Excel). Y a partir de la base de datos que se generó, se elaboraron los gráficos para poder analizar los datos y elaborar las conclusiones. 


\section{Resultados}

A continuación, se presentan los resultados obtenidos por los docentes en las competencias digitales (instrumentales, didáctico-metodológicas y cognitivas); primero se presentan divididos por las instituciones y después los resultados generales.

Figura 1. Resultados de los docentes del Instituto Politécnico Pilar Constanzo con base en la escala de interpretación

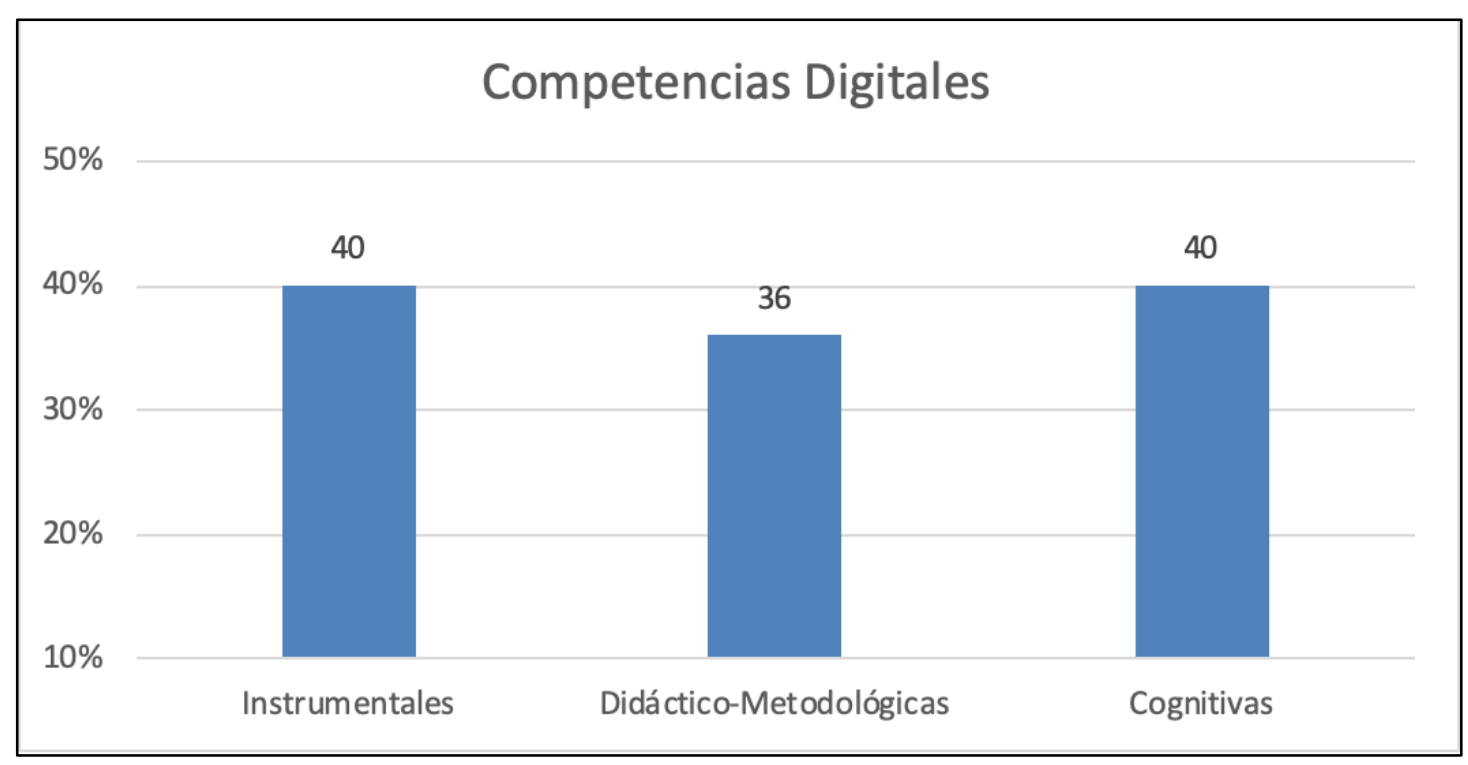

Fuente: Elaboración propia

En la figura 1 se presentan los resultados obtenidos en el Instituto Politécnico Pilar Constanzo. Ahí el cuestionario se aplicó a 77 docentes, quienes obtuvieron promedios de 40 en competencias instrumentales, 36 en didáctico-metodológicas y 40 en competencias cognitivas. Los resultados no difieren mucho en las tres categorías; sin embargo, se muestran mayores deficiencias en las didáctico-metodológicas, las cuales son relevantes para la aplicación de las tecnologías en el aula. 
Figura 2. Resultados de los docentes de la Escuela María Marcia Compres de Vargas con base en la escala de interpretación

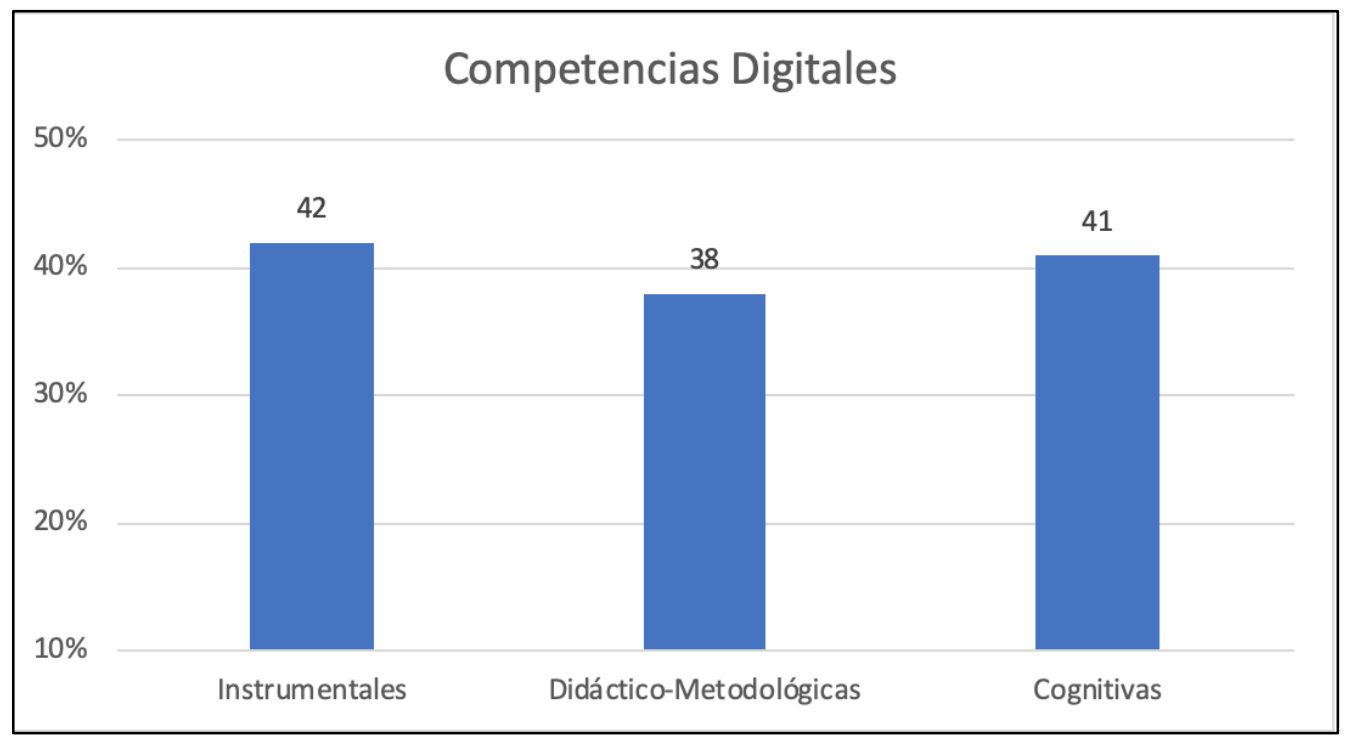

Fuente: Elaboración propia

En la figura 2 se presentan los resultados obtenidos en la Escuela María Marcia Compres de Vargas, donde fueron aplicados 47 cuestionarios a los docentes, quienes obtuvieron promedios de 42 en competencias instrumentales, 38 en didácticometodológicas y 41 en competencias cognitivas. Los docentes obtuvieron los resultados más consistentes en las competencias instrumentales, por lo que se hace referencia al conocimiento de las tecnologías; sin embargo, al igual que en el Instituto Politécnico Pilar Constanzo, los resultados más bajos son en didáctico-metodológicas, referentes al uso de las tecnologías en los procesos de enseñanza y aprendizaje. 
Figura 3. Resultados del total de docentes con base en la escala de interpretación

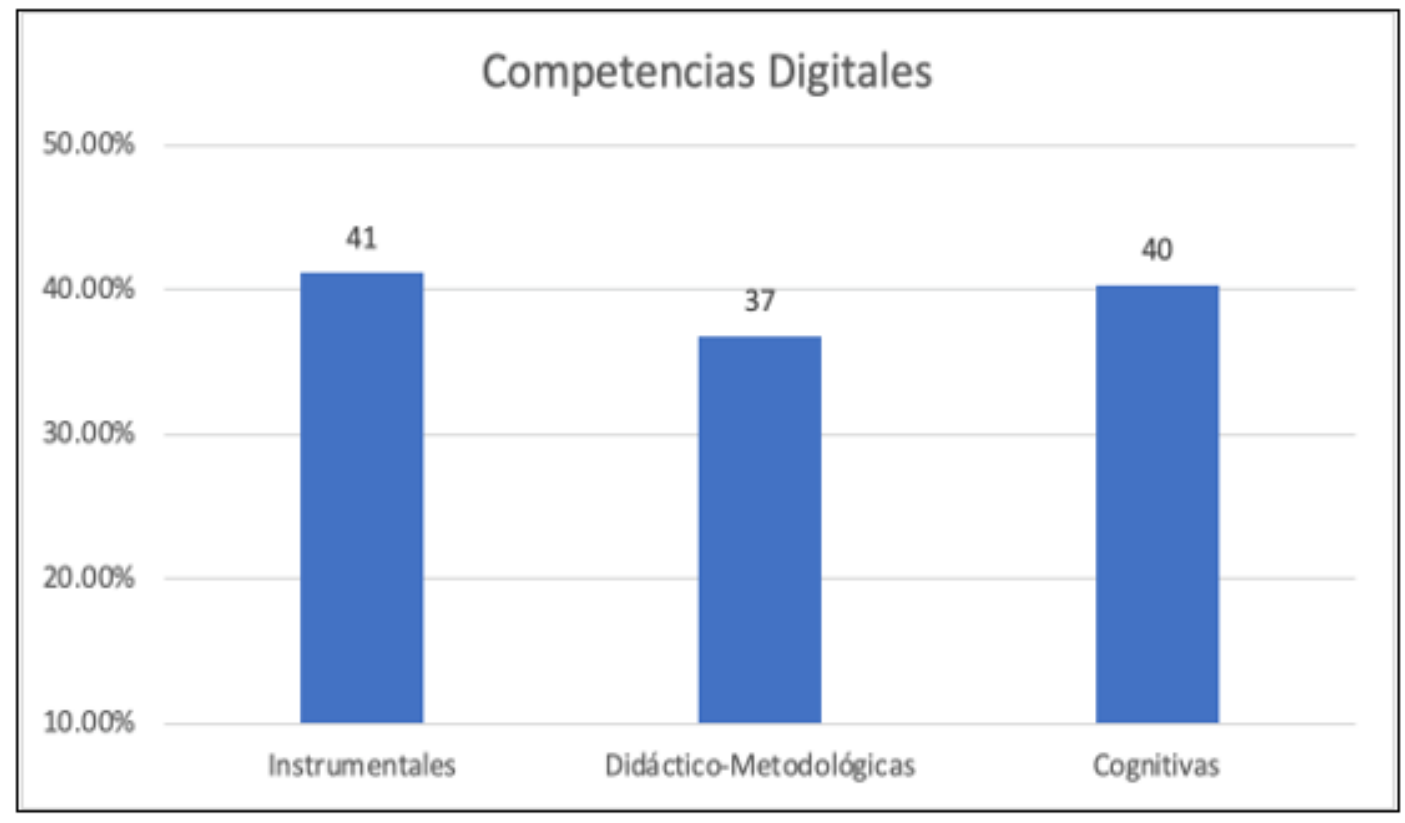

Fuente: Elaboración propia

En la figura 3 se presentan los resultados obtenidos por el total de docentes de las dos instituciones. En conjunto, fueron aplicados 124 cuestionarios a los docentes, quienes obtuvieron promedios de 41 en competencias instrumentales, 37 en didácticometodológicas y 40 en competencias cognitivas. Los resultados muestran las fortalezas y las deficiencias en las competencias digitales. Sin duda se deberá tener como base prioritaria para el diseño de estrategias a las competencias didáctico-metodológicas.

Tabla 4. Resultados de competencias instrumentales del total de docentes

\begin{tabular}{|c|c|c|c|}
\hline \multicolumn{4}{|c|}{ Competencias digitales instrumentales } \\
\hline Categorías & Intervalos & Frecuencia & $\%$ \\
\hline Bueno & $40-50$ & 84 & 68 \\
\hline Regular & $30-39$ & 38 & 30 \\
\hline Deficiente & $<30$ & 2 & 2 \\
\hline Total & & 124 & 100 \\
\hline
\end{tabular}

Fuente: Elaboración propia

En la tabla 4 se pueden observar los resultados obtenidos en las competencias digitales instrumentales por parte de los 124 docentes. Aquí, 84 docentes obtuvieron resultados en la categoría Bueno, lo que representa $68 \%$ del total de la muestra; 38 docentes quedaron en la categoría Regular, es decir, $31 \%$, y por último, solo dos docentes obtuvieron resultados para quedar en la categoría Deficiente, esto es, $2 \%$ del total. Con 
estos datos se puede observar la realidad del desarrollo de competencias instrumentales por parte de los docentes. Es notable que la gran mayoría de los docentes en esta dimensión quedan dentro de la opción Bueno; sin embargo, sumando los resultados de Regular y Deficiente, hay $32 \%$ de docentes con deficiencias, lo cual es igualmente un dato relevante.

Tabla 5. Resultados de competencias didáctico-metodológicas del total de docentes

\begin{tabular}{|c|c|c|c|}
\hline \multicolumn{4}{|c|}{ Competencias digitales didáctico-metodológicas } \\
\hline Categorías & Intervalos & Frecuencia & $\%$ \\
\hline Bueno & $40-50$ & 53 & 43 \\
\hline Regular & $30-39$ & 49 & 39 \\
\hline Deficiente & $<30$ & 22 & 18 \\
\hline Total & & 124 & 100 \\
\hline
\end{tabular}

Fuente: Elaboración propia

En la tabla 5 se pueden observar los resultados obtenidos en las competencias digitales didáctico-metodológicas por parte de los 124 docentes. $\mathrm{Al}$ respecto, 53 docentes obtuvieron resultados en la categoría Bueno, lo que representa $43 \%$ del total de la muestra; 49 docentes quedaron en la categoría Regular, esto es, $39 \%$, y por último, 22 docentes obtuvieron resultados para quedar en Deficiente, $18 \%$ del total. En esta categoría se presentan las mayores deficiencias. Únicamente 53 docentes obtuvieron resultados en la categoría Bueno; sumando los datos de Regular y Deficiente se hace un total de $57 \%$ de docentes con carencias en competencias para el uso de la tecnología con fines educativos.

Tabla 6. Resultados de competencias cognitivas del total de docentes

\begin{tabular}{|c|c|c|c|}
\hline \multicolumn{4}{|c|}{ Competencias digitales cognitivas } \\
\hline Categorías & Intervalos & Frecuencia & $\%$ \\
\hline Bueno & $40-50$ & 75 & 61 \\
\hline Regular & $30-39$ & 40 & 32 \\
\hline Deficiente & $<30$ & 9 & 7 \\
\hline Total & & 124 & 100 \\
& & & \\
\hline
\end{tabular}

Fuente: Elaboración propia 


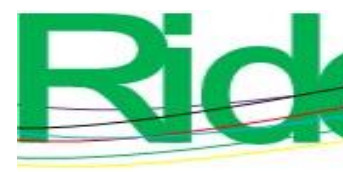

Revista Iberoamericana para la Investigación y el Desarrollo Educativo ISSN 2007 - 7467

En la tabla 6 se pueden observar los resultados obtenidos en las competencias digitales cognitivas por parte de los 124 docentes. En esta dimensión, 75 docentes obtuvieron resultados en la categoría Bueno, esto es, $60 \%$ del total de la muestra; 40 docentes quedaron en la categoría Regular, lo que representa $32 \%$ de la totalidad de los participantes, y por último, nueve docentes obtuvieron resultados para quedar en la categoría Deficiente, esto es, $7 \%$ del total. Como queda en evidencia, la mayoría de los docentes quedaron en la opción Bueno; aun así, sumando las otras dos categorías de interpretación, 39 \% de los docentes requiere de capacitación en esta área. Una vez más, estos datos son relevantes para el diseño de estrategias.

\section{Discusión}

Con ayuda de otros estudios relacionados con la integración de las TIC en el proceso de enseñanza-aprendizaje podemos comparar los resultados obtenidos aquí. Tal es el caso de la investigación llevada a cabo por Espino (2018) en el distrito de Vista Alegre, Perú. Como ya hemos aclarado líneas arriba, Espino (2018) utilizó el mismo instrumento, o mejor dicho, en este estudio se utilizó el mismo instrumento que en el de Espino (2018), quien lo aplicó a 165 docentes. En dicho trabajo, en competencias digitales instrumentales, 146 docentes ( $88 \%$ ) obtuvieron un nivel Bueno y 19 obtuvieron un nivel Regular (12\%), por lo que los obtenidos por nosotros fueron más altos (68 \% de docentes en Bueno, $30 \%$ en Regular y $2 \%$ en Deficiente).

En las competencias digitales didáctico-metodológicas, en Espino (2018) 143 docentes ( $87 \%$ del total) obtuvieron resultados en la categoría Bueno, mientras que 22 (13\%) profesores obtuvieron resultados en la categoría Regular, y no hubo docentes con desarrollo deficiente en este tipo de competencias. En esta dimensión, estos resultados son más consistentes que los obtenidos en el presente estudio, pues aquí $43 \%$ obtuvieron resultados buenos, de acuerdo con la escala de interpretación, $39 \%$ regulares y $18 \%$ deficientes.

En las competencias digitales cognitivas, los docentes del distrito Vista Alegre de Perú (Espino, 2018) obtuvieron los siguientes resultados: 143 docentes (87\% del total) obtuvieron resultados en la categoría Bueno, mientras que $22(13 \%)$ maestros obtuvieron resultados en la categoría Regular, y no hubo participantes con desarrollo deficiente en estas competencias. Por otro lado, en este estudio se obtuvieron los siguientes resultados en la dimensión en cuestión: 61 \% de docentes en la categoría Bueno, 32 \% en la categoría Regular y $7 \%$ en Deficiente. 


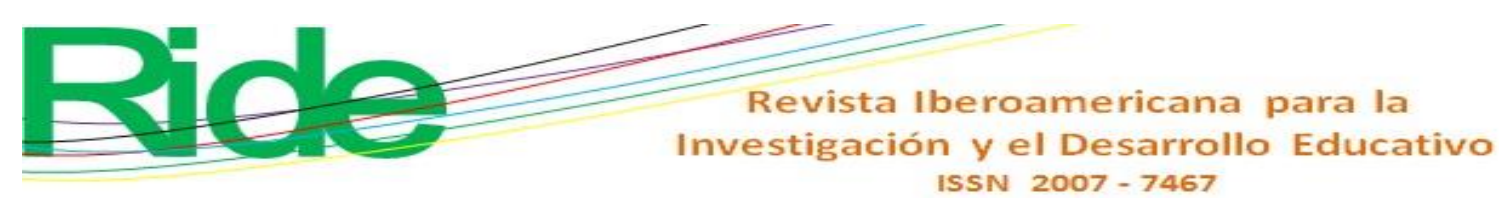

Por tanto, teniendo como referente el estudio efectuado por Espino (2018), se puede concluir que los docentes del distrito Vista Alegre de Perú tienen en general un mayor desarrollo de competencias digitales en comparación con los docentes de los institutos Politécnico Pilar Constanzo y María Marcia Compres de Vargas de nivel secundario del distrito 10-04 de Santo Domingo Este.

Una de las fortalezas de este estudio es la implementación de un cuestionario validado e implementado en una realidad similar, como lo es la realidad latinoamericana, que permite establecer una comparación entre las competencias digitales de los docentes de dos regiones. Otra fortaleza es que este instrumento se aplicó con muy buena consistencia interna entre las dimensiones de las competencias. Asimismo, la muestra utilizada para el estudio conformada por el total de docentes de dos instituciones, que es relevante para la toma de decisiones en estos centros escolares, e incluso para los demás centros del mismo nivel de estudios de Santo Domingo.

El alcance de este proyecto llega únicamente al análisis de las competencias digitales. Una limitación es que no se llegase a concretar un plan de formación basado en este estudio, que correspondería a una siguiente fase de este trabajo. Otra limitación que se puede encontrar es la resistencia de los docentes al cambio tecnológico o la falta de recursos y apoyos para la capacitación docente.

Por otra parte, se considera conveniente aplicar el mismo instrumento a otros centros escolares del mismo nivel de República Dominicana, considerando una muestra probabilística que permita generar un plan de formación y políticas sobre el uso de las TIC a nivel nación en el nivel secundario, ya que la muestra, únicamente dos centros escolares, es una limitante en ese sentido, aunque es relevante para la toma de decisiones en los centros escolares en que se aplicó el instrumento. Por último, se considera también conveniente hacer un análisis de competencias digitales de los estudiantes que permita obtener un panorama más amplio del tema en cuestión. 


\section{Conclusiones}

Los resultados promedio obtenidos en cada institución son muy similares. En ambas se obtuvieron resultados buenos en competencias digitales instrumentales y cognitivas, y regulares en didáctico-metodológicas; por tanto, se puede concluir que las áreas que requieren de mayor atención para contribuir al fortalecimiento del cuerpo de docentes son las didáctico-metodológicas en ambas instituciones.

En competencias digitales instrumentales, 84 de los 124 docentes quedaron en el nivel Bueno, 38 en Regular y dos en Deficiente. Esto significa que hay dos docentes con un nivel de desarrollo muy bajo, quienes, sumados a los otros 38 , constituyen un total de 40 docentes que requieren capacitación para alcanzar el nivel óptimo en esta dimensión.

En la dimensión de competencias digitales didáctico-metodológicas, 53 docentes quedaron en la categoría de Bueno, de acuerdo con la escala de interpretación, mientras que 49 obtuvieron resultados en la categoría de Regular, y 22 en Deficiente, con un muy bajo nivel de desarrollo de competencias. Por tanto, en esta categoría se concluye que 71 docentes requieren de capacitación para arribar al nivel óptimo de competencias didáctico-metodológicas.

Respecto a la dimensión de competencias digitales cognitivas, 75 docentes obtuvieron resultados en la categoría de Bueno, mientras que 40 obtuvieron un resultado Regular y nueve obtuvieron Deficiente. Se concluye que en esta categoría 49 docentes requieren de capacitación para adquirir el nivel óptimo de desarrollo de competencias cognitivas.

Para obtener mejores resultados en el desarrollo de competencias digitales por parte de los docentes de este estudio, y para obtener los beneficios de su implementación en el aula, se considera conveniente desarrollar un plan de formación continua dirigido a la alfabetización tecnológica de las diversas herramientas, con el objetivo de orientar, formar, capacitar y profesionalizar al personal docente. Este estudio es relevante ya que permite tener un referente de los niveles de desarrollo de competencias de los docentes, y también es valioso para identificar las áreas prioritarias para el plan de formación.

Los resultados de este estudio establecen como prioridad para un plan de formación las competencias digitales didáctico-metodológicas, donde los participantes obtuvieron los resultados más bajos. Asimismo, se considera conveniente utilizar los resultados de este estudio para la estructuración de los contenidos del plan de formación, considerando tanto la conceptualización de las competencias como los indicadores que se estuvieron analizando en el estudio. Esto a razón de que se puede concluir que un 
porcentaje mayor a $50 \%$ de los docentes a quienes se les aplicó el cuestionario requieren capacitación para adquirir el nivel óptimo de desarrollo de competencias digitales en alguna dimensión.

Por último, se sugiere el establecimiento de políticas institucionales para el uso de las TIC que contemplen el plan de formación antes mencionado, con la finalidad no únicamente de desarrollar competencias requeridas, sino también de una actualización continua y permanente que les permita a los docentes estar al día en el uso de los recursos tecnológicos, y adaptarse a los cambios constantes y actualizaciones de estos, y así aprovechar al máximo sus ventajas y potencialidades para la mejora de los procesos de enseñanza y aprendizaje.

\section{Referencias}

Alonso, C., Casablancas, S., Domingo, L., Guitert, M., Moltó, O., Sánchez, J. A. y Sancho, J. (2010). De las propuestas de la Administración a las prácticas del aula. Revista de Educación, (352), 53-76. Recuperado de https://pdfs.semanticscholar.org/a2d7/ddcf934891cc17476c9fc72ab0ae290f582d .pdf.

Ayala, F. (2018). El trabajo docente mediado con tecnología de la información y comunicación en la telesecundaria. Representaciones sociales de profesores. RIDE Revista Iberoamericana para la Investigación y el Desarrollo Educativo, 8(16), 557-579. Recuperado de https://doi.org/10.23913/ride.v8i16.358.

Cabero, J., Arancibia, M., Valdivia, I. y Aranedas, S. (2018). Percepciones de profesores y estudiantes de la formación virtual y de las herramientas en ellas utilizadas. Revista Diálogo Educacional, 18(56), 149-163. Recuperado de http://dx.doi.org/10.7213/1981-416X.18.056.DS07.

Celina, H. y Campo, A. (2005). Aproximación al uso del coeficiente alfa de Cronbach. Revista Colombiana de Psiquiatría, 34(4), 572-580. Recuperado de https://www.redalyc.org/pdf/806/80634409.pdf.

Christian, S. and Mathrani, A. (2014). ICT Education: Socio-Learning Issues Faced by International Students. Paper presented at the $35^{\text {th }}$ International Conference on Information Systems. Auckland, 14-17 December 2014.

Christensen, C., Johnson, C. and Horn, M. (2008). Disrupting Class, Expanded Edition: How Disruptive Innovation Will Change the Way the World Learns. United States: McGraw-Hill Education. 
Comisión Europea. (30 de diciembre de 2006). Recomendación del Parlamento Europeo y del Consejo de 18 de diciembre de 2006 sobre las competencias clave para el aprendizaje permanente. Diario Oficial de la Unión Europea.

Enguita, M. (2016). La educación en la encrucijada. Madrid, España: Fundación Santillana.

Espino, J. (2018). Competencias digitales de los docentes y desempeño pedagógico en el aula. (tesis de maestría). Universidad de San Martín de Porres, Lima. Recuperado de http://repositorio.usmp.edu.pe/handle/usmp/4525.

Fernández, F. y Fernández, M. J. (2016). Los docentes de la Generación Z y sus competencias digitales. Comunicar, 46(24), 97-105. Recuperado de http://ddfv.ufv.es/handle/10641/1177.

Franky, A. y Chiappe, A. (2018). Familias de educación en el hogar de ICT: un estudio de caso cualitativo múltiple. Ensaio: Avaliação e Políticas Públicas em Educação, 26(101), 24-46. Recuperado de https://doi.org/10.1590/s010440362018002601507.

Jiménez, M. y Espejel, M. (2019). Análisis de aspectos tecnológicos y educativos que influyen en el ingreso económico en México. RIDE Revista Iberoamericana para la Investigación y el Desarrollo Educativo, 10(19), 1-20. Recuperado de https://doi.org/10.23913/ride.v10i19.489.

Lucero, I. y Meza, S. (2012). Validación de instrumentos para medir conocimientos. Recuperado de https://nanopdf.com/download/validacion-de-instrumentos-paramedir-conocimientos_pdf.

Medina, A. y Ballano, S. (2015). Retos y problemáticas de la introducción de la educación mediática en los centros de secundaria. Revista de Educación, (369), 135-158.

Prensky, M. (2012). From Digital Natives to Digital Wisdom: Hopeful Essays for 21st Century Learning. United States: Corwin Press.

Quintana, J. (2000). Competencias en tecnologías de la información del profesorado de educación infantil y primaria. Revista Interuniversitaria de Tecnología, 3(25), $166-174$ Recuperado de http://www.ub.edu/ntae/jquintana/articles/competicformprof.pdf.

Reis, V. e Lunardi, G. (2018). De iniciantes a vanguardias: o uso de tecnologías digitais jovens professores. Holos, 34(1), 297-316. Recuperado de http://www2.ifrn.edu.br/ojs/index.php/HOLOS/article/view/4867.

Rincón, C. (2018). Proceso de transferencia en el uso de las TIC en las escuelas normales del estado de Zacatecas. RIDE Revista Iberoamericana para la Investigación y el 
Desarrollo Educativo, 8(16), 1-25. Recuperado de https://doi.org/10.23913/ride.v8i16.361.

Rubilar, P., Alveal, F. y Fuentes, A. (2017). Evaluación de la alfabetización digital y pedagógica en TIC, a partir de las opiniones de estudiantes en Formación Inicial Docente. Educação e Pesquisa, 43(1), 127-143. Recuperado de http://dx.doi.org/10.1590/s1517-9702201701154907.

Silveira, M. y de Luca, N. (2015). Política educativa y cultura digital: entre las prácticas escolares y las prácticas sociales. Perspectiva, Florianópolis, 33(2), 499521. Recuperado

de researchgate.net/publication/318843911_Politica_educativa_e_cultura_digital_e ntre_praticas_escolares_e_praticas_sociais/link/59813b670f7e9b7b5245aadc/do wnload.

Sosa, O. y Manzuoli, C. (2019). Modelos para la integración pedagógica de las tecnologías de la información y la comunicación: una revisión de la literatura. Ensaio: Avaliação e Políticas Públicas em Educação, 27(102), 12956. Recuperado de https://doi.org/10.1590/s0104-40362018002701720.

Turra, O y Flores, C. (2018). Formación práctica desde la vocación del estudiante de pedagogía. Ensaio: Avaliação e Políticas Públicas em Educação, 27(103), 385405. Doi: http://dx.doi.org/10.1590/s0104-40362018002601517

Zabala, A. (2000). Práctica educativa: cómo enseñar. Barcelona, España: Grao. 


\begin{tabular}{|l|l|}
\hline Rol de Contribución & Autor (es) \\
\hline Conceptualización & José Antonio Volquez Pérez \\
\hline Metodología & $\begin{array}{l}\text { José Antonio Volquez Pérez (Principal) } \\
\text { Carlos Miguel Amador Ortíz (apoyo) }\end{array}$ \\
\hline Software & Carlos Miguel Amador Ortíz \\
\hline Validación & Carlos Miguel Amador Ortíz \\
\hline Análisis Formal & $\begin{array}{l}\text { José Antonio Volquez Pérez (Principal) } \\
\text { Carlos Miguel Amador Ortíz (apoyo) }\end{array}$ \\
\hline Investigación & José Antonio Volquez Pérez \\
\hline Recursos & José Antonio Volquez Pérez \\
\hline Curación de datos & $\begin{array}{l}\text { José Antonio Volquez Pérez (Principal) } \\
\text { Carlos Miguel Amador Ortíz (apoyo) }\end{array}$ \\
\hline $\begin{array}{l}\text { Escritura - Preparación del } \\
\text { borrador original }\end{array}$ & José Antonio Volquez Pérez \\
\hline $\begin{array}{l}\text { Escritura - Revisión } \\
\text { edición }\end{array}$ & Carlos Miguel Amador Ortíz \\
\hline Visualización & José Antonio Volquez Pérez \\
\hline Supervisión & José Antonio Volquez Pérez \\
\hline Administración & José Antonio Volquez Pérez \\
\hline
\end{tabular}

